\title{
B physics with ATLAS and CMS
}

\section{Brigitte EPP*}

University of Innsbruck, Innstruck

E-mail: brigitte.epp@cern.ch

For ATLAS and CMS collaborations

The B-physics programs of the ATLAS and CMS collaborations cover a wide range of the different periods at the LHC. The expected $b \bar{b}$ cross-section allows sensitive tests of QCD predictions of production in proton-proton collisions at the LHC and high precision measurements as a test of the Standard Model. B-physics searches and indirect constraints from B decays will provide information on new physics phenomena.

Physics at LHC 2008

29 September - October 4, 2008

Split, Croatia

${ }^{*}$ Speaker. 


\section{Introduction}

From a total cross-section of about $100 \mathrm{mb}$ at the Large Hadron Collider (LHC), operating as a proton-proton collider at a center-of-mass energy of $14 \mathrm{TeV}$, one expects roughly $500 \mu \mathrm{b}$ for $b \bar{b}$-pair production corresponding to $2 \times 10^{12} b \bar{b}$ events per year at a luminosity $L=10^{33} \mathrm{~cm}^{-2} \mathrm{~s}^{-1}$. Although ATLAS [1] and CMS [2] are general purpose detectors, B-physics requirements were taken into account in the detector and trigger building phases. Compared to earlier accelerators the higher muon rate from B-events will allow the muon and di-muon trigger strategies to collect a huge number of events of the B-hadron species.

The B-physics programs of ATLAS and CMS cover a wide range of measurements on Standard Model (SM) and new physics effects over the different LHC periods. During the first part of the early data period with an expected integrated luminosity of $L_{\text {int }}=10-100 \mathrm{pb}^{-1}$, the understanding of detector properties and the muon triggers will have first priority. Early measurements of $J / \psi$ and $\Upsilon$ and the $b$-quark production cross-sections will provide a good testbed for various QCD models at the new energy. The large $b$-quark cross-section will allow the early extraction of exclusive decays, like $B^{+} \rightarrow J / \psi K^{+}, B_{d}^{0} \rightarrow J / \psi K_{S}^{0}$ and $B_{S} \rightarrow J / \psi \phi$, which will be a tester for detector calibration. During the data collection period of $L_{\text {int }}=200 \mathrm{pb}^{-1}-1 \mathrm{fb}^{-1}$, the high statistics will allow to improve the world precision of lifetime measurements, set a new limit for $B_{S} \rightarrow \mu \mu$ and improve measurement on $B_{C}$ and $\Lambda_{b}$. The main data period for B-physics studies will cover the range of $L_{i n t}=10-30 \mathrm{fb}^{-1}$ and higher. Exclusive rare decays, strongly suppressed in the SM, can be analyzed in the purely muonic channel $B_{S} \rightarrow \mu \mu$ and semi-muonic channels, like $\mathrm{b} \rightarrow \mathrm{d}$,s $\mu \mu$. Measurement of the $\Lambda_{b}$ polarization in the channel $\Lambda_{b} \rightarrow \Lambda J / \psi$ and CP-violation and $B_{S}$ mixing studies using decays $B_{S} \rightarrow J / \psi \phi$ and $B_{S} \rightarrow D_{S} \pi\left(a_{1}\right)$ will contribute to test the SM and search for new physics. Constraints on the lepton-flavor-violating phenomenon using the channel $\tau \rightarrow \mu \mu \mu$ can be provided through the determination of the respective branching ratio. For the high luminosity period of $10^{34} \mathrm{~cm}^{-2} \mathrm{~s}^{-1}$ the rare decays of $b$-hadrons involving muon pairs are feasible and their studies will therefore be continued after the periods of low luminosity.

\section{Triggers}

ATLAS and CMS use a multi-level trigger system to reduce the event rate from the bunchcrossing rate of $40 \mathrm{MHz}$ to a total rate of $\sim 100-200 \mathrm{~Hz}$. ATLAS has opted for a three-level trigger: Level 1 (L1), implemented in hardware, selects events based on information from the calorimeters and muon trigger chambers and identifies Regions of Interest (RoI) for further processing. The Level 2 trigger (L2) has access to all event data, with full precision and granularity. L2 uses L1 RoIs to confirm L1 trigger selections with emphasis on fast rejection and fast algorithms including measurements in the Inner Detector (ID). The third level, called Event Filter (EF), refines L2 selection, using L2 RoIs and offline-like algorithms and can access detailed alignment and calibration data. L2 and EF are software triggers; together they form the ATLAS High Level Trigger (HLT). The trigger for B-Physics, accounted for $5-10 \%$ of the total trigger resources in ATLAS, must be fast, efficient and selective. Depending on the instantaneous luminosity single muon trigger thresholds from $p_{T, \mu}>4 \mathrm{GeV}$ upwards together with JET- or an electromagnetic RoI and di-muon $p_{T}$ thresholds from $p_{T, \mu}>4 \mathrm{GeV}$ rising to $p_{T, \mu}>6 \mathrm{GeV}$ for $10^{33} \mathrm{~cm}^{-2} \mathrm{~s}^{-1}$ will be 
employed at L1. At L2 there are two approaches to select di-muon events. The first approach (topological trigger) is to start from a di-muon trigger at L1, which produces two muon RoI. The reconstruction of a muon is confirmed separately in each of these RoI. An alternative approach (TrigDiMuon algorithm) is to start with a L1 single muon trigger and search for two muons in an enlarged RoI around this trigger muon. Tracks are reconstructed in the ID and extrapolated to the muon spectrometer to tag muon tracks. Depending on the luminosity two different strategies are used for finding tracks in the ID. Full reconstruction of the whole ID can be performed at $10^{31}$ $\mathrm{cm}^{-2} \mathrm{~s}^{-1}$. At higher luminosities, reconstruction will be limited to RoI defined by the $\mathrm{L} 1$ calorimeter jets. For hadronic final states like $B_{S} \rightarrow D_{S} \pi\left(a_{1}\right)$ ID tracks are combined to reconstruct first the $\phi \rightarrow K^{+} K^{-}$, then the $D_{S} \rightarrow \phi \pi$. More information on ATLAS B-physics trigger topics can be found in reference [3].

The CMS trigger system (see reference [4]) uses a twofold strategy and has merged the software based levels two and three in a single processor farm, called High Level Trigger (HLT). L1 is a hardware trigger, using coarse information from the calorimeter and muon detector. The HLT has access to all event data, with full precision and granularity and can use the offline software. Two concepts are followed: regional seed generation limits track seeding to specific RoIs, e.g. a cone around the refined L1 muon candidate direction. Already with six reconstructed hits both the efficiency and resolution are comparable to the full tracking performance. The second concept follows partial reconstruction, which means reconstruction is stopped after some criteria are met, e.g. a resolution of $2 \%$ on the transverse momentum. For the B-physics trigger in CMS the threshold for inclusive isolated single muons is at $p_{T ; \mu}>7 \mathrm{GeV}$ (14 GeV for high luminosity), for dimuon events both muons must have $p_{T, \mu}>3 \mathrm{GeV}$ ( $7 \mathrm{GeV}$ for high luminosity). In the HLT exclusive $b$-decays are reconstructed around the L1 muon using the partial track reconstruction.

\section{Quarkonium production and polarization}

Due to the high production rate of quarkonia, such as $J / \psi$ and $\Upsilon$, and the reasonable branching fraction into charged leptons, one of the first physics results with early data at the LHC will come from quarkonium states. The narrow resonances can be used as tools for alignment and calibration of the trigger, tracking and muon system. On the other hand the understanding of the prompt onia production mechanism is a good testbed for various QCD calculations and already with an integrated luminosity of $10 \mathrm{pb}^{-1}$ there will be enough statistics to probe different production models. For CMS the dimuon mass spectrum including background and signal is shown in Figure 1. The measurement of the differential $J / \psi$ cross section as shown in Figure 2 is feasible already for an integrated luminosity of $3 \mathrm{pb}^{-1}$. More information is given in reference [5].

Figure 3 shows the invariant mass distribution in the $J / \psi$ and $\Upsilon$ region for the di-muon dataset for ATLAS. Background suppression to select direct $J / \psi$ events is achieved through vertexing and impact parameter cuts on the muons and a pseudo-proper time cut on the reconstructed quarkonium candidate [3]. In order to extract the $J / \psi$ and $\Upsilon$ polarizations $\alpha=\left(\sigma_{T}-2 \sigma_{L}\right) /\left(\sigma_{T}+2 \sigma_{L}\right)$ the simulated data are fitted to the acceptance-corrected $\cos \theta^{*}$ distributions, where $\theta^{*}$ specifies the polarization angle of the $\mu^{+}$w.r.t. the quarkonium momentum in the rest frame of the quarkonium. This quantity serves as an important measurement for discriminating different production models. The different polarization states can be distinguished well as shown in Figure 4 for the region 


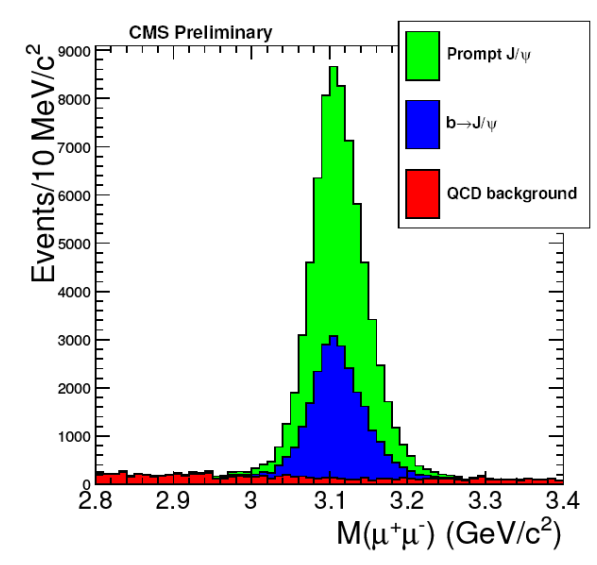

Figure 1: Dimuon invariant mass distribution normalized to $3 \mathrm{pb}^{-1}$. The green (light grey), blue (black) and red (dark grey) areas are the prompt, non-prompt and QCD background contributions, respectively.

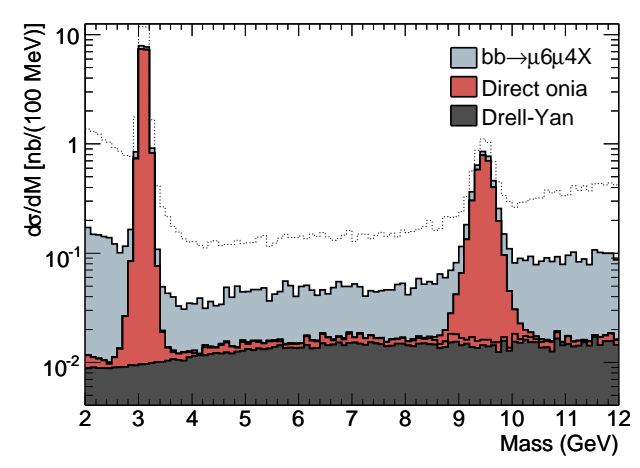

Figure 3: Sources of low invariant mass di-muons, after background suppression cuts in ATLAS. The light dotted line shows the background level before vertexing and proper-time cuts.

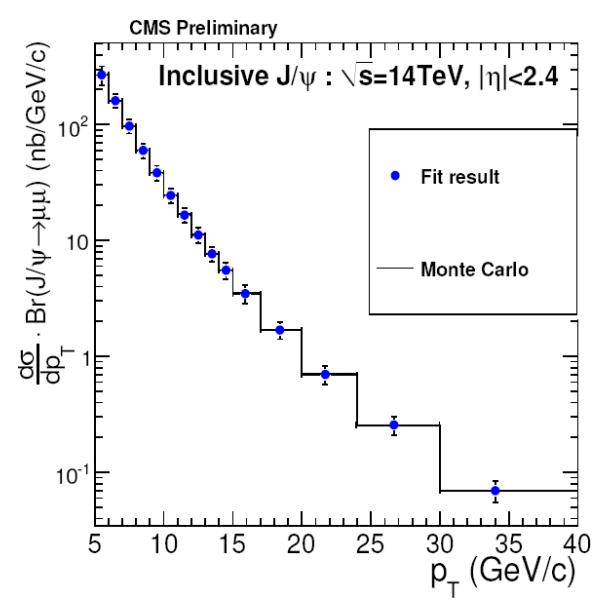

Figure 2: The inclusive $J / \psi$ differential cross section, $\mathrm{d} \sigma / \mathrm{d} p_{T}$ as a function of $p_{T}^{J / \psi}$, integrated over the pseudorapidity range $\left|\eta^{J / \psi}\right|<2.4$, corresponding to an integrated luminosity of $3 \mathrm{pb}^{-1}$.

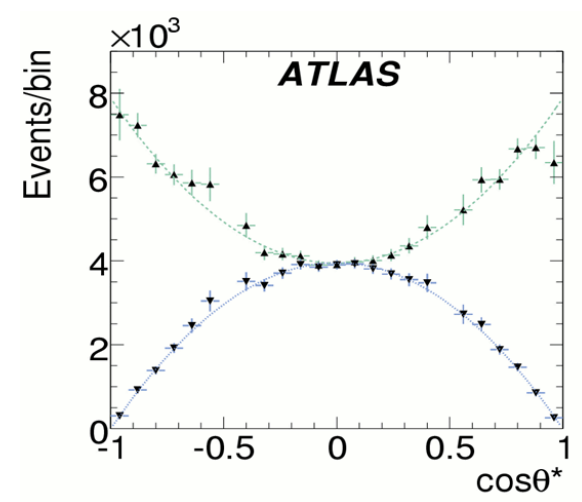

Figure 4: Acceptance and efficiency corrected $J / \psi$ polarization angle distribution $\left(\alpha_{g e n}=\mp 1\right)$ for $13 \mathrm{GeV}<p_{T, J / \psi}<15 \mathrm{GeV}$.

$13 \mathrm{GeV}<p_{T, J / \psi}<15 \mathrm{GeV}$. With $10 \mathrm{pb}^{-1}$, Atlas expects to measure the polarization of $J / \psi$ with the precision of the order of 0.02-0.06, depending on the level of polarization itself, in a wide range of transverse momenta, $p_{T}$ between 10 and $20 \mathrm{GeV}$ and beyond. In case of $\Upsilon$, the expected precision is somewhat lower, of the order of 0.20 .

\section{CP Violation and other parameters of $B_{S} \rightarrow J / \psi \phi$}

The weak mixing phase $\Phi_{S}$ of the $B_{S}$-meson is very small and precisely predicted within the SM, but new physics models predict larger $\Phi_{S}$. The decay $B_{S} \rightarrow J / \psi \phi$ is one of the most promising channels at the LHC with respect to the measurement of $\Phi_{S}$ and the decay width difference $\Delta \Gamma_{S}$. Due to the efficient di-muon trigger and the good background suppression possibilities ATLAS and 


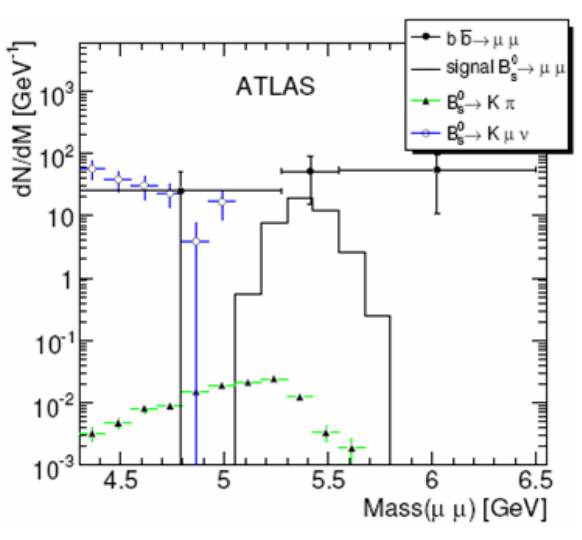

Figure 5: Di-muonic invariant mass spectrum of the signal B-hadrons, inclusive $b \bar{b}$ contribution and selected misidentification-based background channels for ATLAS. Statistics corresponds to $L_{i n t}=10 \mathrm{fb}^{-1}$.

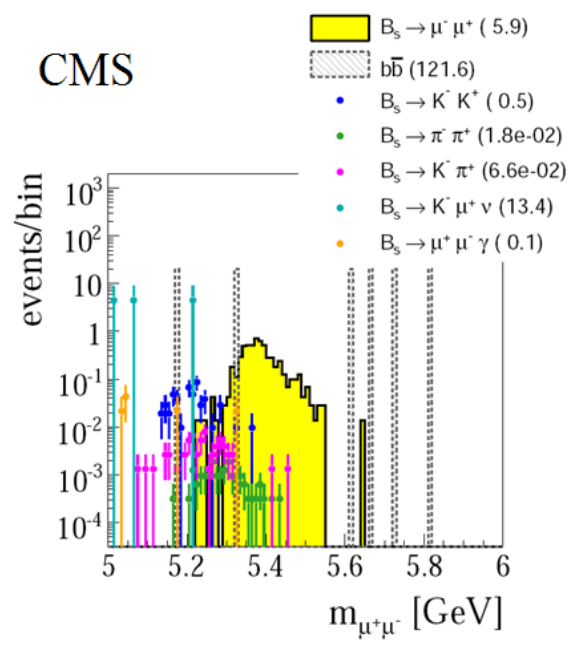

Figure 6: Di-muonic invariant mass spectrum after selections for $L_{i n t}=10 \mathrm{fb}^{-1}$ also showing rare background contributions.

CMS are expected to produce enough statistics of $B_{S} \rightarrow J / \psi \phi$ events per year to confirm or reject possible contributions beyond the SM, which may alter CP violation in B-decays. Simulation of the time dependent three-dimensional angular distribution of the final state proved that both experiments can perform a model independent determination of $\Phi_{S}$ and $\Delta \Gamma_{S}$. A multivariable analysis with experimental information on three angles, $B_{S}$ decay time resolution, flavor tag quality, signal statistics, background contributions and the oscillation frequency $\Delta m_{S}$ will determine the sensitivity.

\section{Sensitivity in $B_{S} \rightarrow \mu^{+} \mu^{-}$}

The di-muonic rare decay $B_{S} \rightarrow \mu^{+} \mu^{-}$can occur in the SM through higher order diagrams of flavor-changing-neutral currents and transitions are also helicity suppressed, which results in a very low branching ratio (BR) of $(3.42 \pm 0.52) \times 10^{-9}$ [6]. The special motivation to analyze this decay channel is the fact, that the low BR gives room for new physics effects, which may enhance the value by several orders of magnitude through new loop diagrams. The present best limit is set by Tevatron measurements of $\mathrm{CDF}$, at $2 \mathrm{fb}^{-1}$ of data, setting an upper limit on the BR to $5.8 \times 10^{-8}$ at $95 \%$ CL [7]. For the analysis ATLAS and CMS have similar discriminating variables using different selection cut values, like the isolation of the muon pair, the significance of the decay length, the angle between the di-muon momentum and direction to the primary vertex and a mass window around the invariant mass of the $B_{S}$, which is shown in Figure 5 for ATLAS and Figure 6 for CMS, respectively.

The key issue for the $B_{S} \rightarrow \mu^{+} \mu^{-}$discovery at the LHC is the suppression of the backgrounds, basically combinatorial background, whereas contributions from other sources like $B_{S} \rightarrow K^{-} \mu^{+} v$, $B_{d} \rightarrow K^{-} \pi^{+}$and $B_{d} \rightarrow \pi^{+} \pi^{-}$are found to be negligible. See references [3], [8] concerning ATLAS and CMS for detailed information on analysis and expected results. Assuming the Standard 
Model, ATLAS expects a signal of 5.7 events with a background of $14_{-10}^{+13} b \bar{b} \rightarrow \mu^{+} \mu^{-} X$ events for an integrated luminosity of $10 \mathrm{fb}^{-1}$, respectively CMS will yield 6.1 signal events with a background contribution of $14_{-14}^{+22}$. It is evident that the background uncertainties are large due to limited statistics on simulated events. For $2 \mathrm{fb}^{-1}$ ATLAS and CMS will achieve a sensitivity on the BR $<10^{-8}$ and reach the SM prediction region with $10-20 \mathrm{fb}^{-1}$. After three years at a luminosity of $10^{33} \mathrm{~cm}^{-2} \mathrm{~s}^{-1}$ a $3 \sigma$ evidence on reconstructing the $B_{S} \rightarrow \mu^{+} \mu^{-}$signal will be obtained. The strategy is to continue the di-muon programme with the nominal LHC luminosity yielding in a $5 \sigma$ observation after one year of data taking at $10^{34} \mathrm{~cm}^{-2} \mathrm{~s}^{-1}$.

\section{Exclusive semi-leptonic decays}

$b \rightarrow s(d) l^{+} l^{-}$flavor-changing-neutral current transitions are only induced at the one-loop level in the SM leading to small branching fractions. This opens room to provide a good test of the SM as well as indirect search for contributions from models beyond the SM. ATLAS is capable to reconstruct semi-rare decays of all B-hadrons $B^{+}, B^{0}, B_{S}$ and $\Lambda_{b}$ with specific requirements for the HLT on a track search for charged hadrons coming from these decays. Ongoing studies show that ATLAS and CMS have potential to achieve the sensitivity to distinguish between the SM and certain classes of new physics models.

\section{Lepton Flavor Violation $\tau \rightarrow \mu \mu \mu$}

Lepton-flavor violation LFV in the SM is negligible, but some new physics models allow branching ratios of the order of $10^{-10}-10^{-7}$. The present $90 \% \mathrm{CL}$ upper limit on $\mathrm{BR}(\tau \rightarrow \mu \mu \mu)$ is $3.2 \times 10^{-8}$ [9]. Out of the number of LFV $\tau$-decays, the channel $\tau \rightarrow \mu \mu \mu$ is likely to be the only detectable at ATLAS and CMS, although studies of the other channels are also under consideration. CMS has performed a detailed study of this particular $\tau$-decay channel [8] considering three different $\tau$ sources at LHC, which have a clean experimental signature and a suitable trigger, $W \rightarrow \tau \nu, Z \rightarrow \tau \tau$ and $b$-quark meson decays $B \rightarrow \tau X$. An expected sensitivity of the order of $10^{-8}$ on the $\operatorname{BR}(\tau \rightarrow \mu \mu \mu)$ for $30 \mathrm{fb}^{-1}$ is estimated. Figure 7 shows the invariant mass $\tau \rightarrow \mu \mu \mu$ fitted with a single Gaussian function giving a resolution of $24 \mathrm{MeV} / \mathrm{c}^{2}$.

\section{Conclusion}

ATLAS and CMS B-physics programs are prepared for all LHC luminosities, aiming to collect valuable B-physics data in order to test Standard Model and to constrain new physics. 


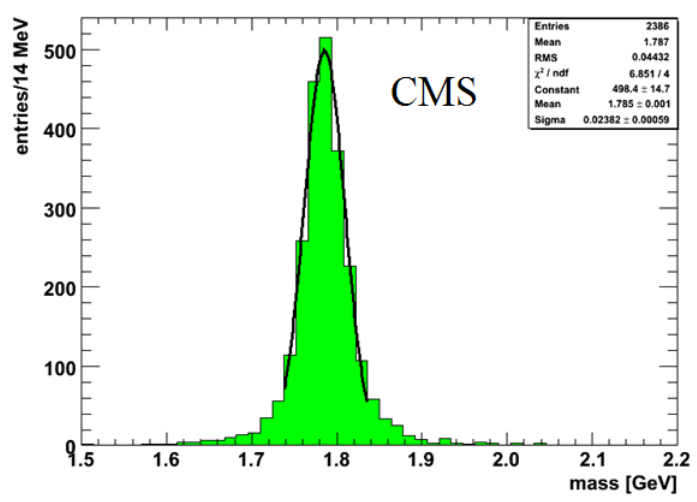

Figure 7: Invariant mass of the decay $\tau \rightarrow \mu \mu \mu$ with $10 \mathrm{fb}^{-1}$ for CMS.

\section{References}

[1] The ATLAS Collaboration, The ATLAS Experiment at the CERN Large Hadron Collider, JINST 3 S08003, 2008.

[2] The CMS Collaboration, The CMS experiment at the CERN LHC, JINST 3 S08004, [2008].

[3] The ATLAS Collaboration, Expected Performance of the ATLAS Experiment Detector, Trigger, Physics, CERN-OPEN-2008-020, Geneva, (2008) [arXiv:0901.0512v1].

[4] The CMS Collaboration, CMS physics : Technical Design Report, CERN-LHCC-2006-021, [2006].

[5] The CMS Collaboration, Feasibility study of a J/ $\psi$ cross section measurement with early CMS data, CMS PAS BPH-07-002, 2008.

[6] A.J.Buras et al, $B \rightarrow \pi \pi$, New Physics in $B \rightarrow \pi K$ and Implications for Rare $K$ and $B$ Decays, [arXiv:hep-ph/0312259v2,2004].

[7] The CDF Collaboration, Search for $B_{S} \rightarrow \mu^{+} \mu^{-}$and $B_{d} \rightarrow \mu^{+} \mu^{-}$Decays with $2 \mathrm{fb}^{-1}$ of $\bar{p}$ Collisions, Phys.Rev.Lett.100:101802, [arXiv:0712.1708v2].

[8] A.Starodumov, M.Biasini, Flavor Physics with CMS at LHC, CMS CR-2008/070.

[9] C.Amsler et al. [Particle Data Group], Phys. Lett.B 667 (2008). 